\title{
Applicazione della meccanica della frattura viscoelastica alla previsione della vita di tubi in polibutene
}

\author{
Luca Andena, Marta Rink, Roberto Frassine \\ Politecnico di Milano, Dipartimento di Chimica, Materiali e Ingegneria Chimica "G. Natta”
}

RIASSUNTO. Il Polibutene-1 isotattico (i-PB1) è un materiale polimerico usato per la produzione di tubi per il trasporto di fluidi in pressione. In questo lavoro si sono studiati due tipi di i-PB1 prodotti da Basell che differiscono per grado di isotatticità.

Si sono condotte prove di frattura a diverse temperature e velocità di spostamento imposte. Si è utilizzata una configurazione di flessione su provini con singolo intaglio (SENB) unitamente a quella di doppia trave a sbalzo (DCB), quest'ultima limitatamente allo studio della fase di propagazione. Al fine di individuare con precisione l'innesco della frattura e la velocità di propagazione della stessa si è fatto ricorso a metodi ottici.

Dal punto di vista fenomenologico durante la propagazione si assiste alla formazione di zone in cui il materiale risulta fortemente stirato. La frattura in esse avanza con una lacerazione continua che si alterna a salti repentini in occasione del brusco cedimento di queste zone, associato a conseguenti cadute del carico. Questa parziale instabilità è stata osservata sui due materiali per entrambe le configurazioni di prova.

I risultati ottenuti sono stati interpretati seguendo l'approccio della meccanica della frattura e applicando uno schema di riduzione di tipo tempo-temperatura che ha permesso di descrivere il comportamento viscoelastico del materiale su un intervallo temporale di diverse decadi.

I risultati hanno permesso di applicare un modello analitico per la previsione della vita utile di tubi in pressione. Il modello si è mostrato in buon accordo con i dati sperimentali disponibili da prove condotte su tubi dello stesso materiale.

ABSTRACT. Isotactic polybutene-1 (i-PB1) is a polymer used for the manufacturing of pressurized pipes. In this work two grades of i-PB1 with a different degree of isotacticity have been investigated; they have been supplied by Basell Polyolefins.

Fracture tests have been performed at various temperatures and testing speeds. Two configurations have been used, single edge notch bending (SENB) and double cantilever beam (DCB), the latter only to study crack propagation. Optical methods have been used to detect crack initiation and measure propagation speed.

From the phenomenological point of view, the formation of highly stretched material regions has been observed during crack propagation. A continuous tearing of these regions as the crack advances has often been interrupted by their sudden rupture, with the load decreasing accordingly. This partial instability has been observed on both material grades, with both testing configurations.

Results of the tests have been interpreted using the fracture mechanics framework; a time-temperature superposition scheme has been adopted to represent viscoelastic behavior over several decades. An analytical model has been applied to predict the lifetime of pressurized pipes. A good agreement has been reported between model predictions and experimental data obtained from tests on polybutene pipes.

PAROLE CHIAVE. Polibutene; frattura; viscoelasticità; polimero

\section{INTRODUZIONE}

Il Polibutene-1 isotattico (i-PB1) viene sempre più frequentemente usato come materiale per la fabbricazione di tubi per applicazioni quali riscaldamento e distribuzione di acqua potabile. Secondo la Polybutene Piping Systems Association (PBPSA), l'utilizzo dell'i-PB1 comporta diversi vantaggi rispetto ai concorrenti più tradizionali, ad esempio per quanto riguarda le sue proprietà meccaniche nel lungo periodo ed il comportamento a temperature elevate. Vi sono studi approfonditi che riguardano la transi- zione dell'i-PB1 tra le sue due forme cristalline (denominate I e II) in seguito alla fusione [1, 2]. Di contro le proprietà meccaniche dell'i-PB1 non sono state finora oggetto di grande attenzione in letteratura.

Un fenomeno di sicuro interesse per i tubi in pressione in materiale polimerico è quello dell'innesco e successiva propagazione lenta di cricche a partire da difetti superficiali. La resistenza del materiale a questo tipo di cedimento può essere caratterizzata mediante prove accelerate su tubi in pressione ad elevata temperatura. Un andamento tipico è riportato schematicamente in Fig. 1. Si distin- 
guono due regioni: a valori di sforzo (e quindi pressione) elevati si assiste al cedimento duttile della sezione resistente del tubo mentre per valori inferiori si ha il campo effettivamente interessato dalla frattura per propagazione lenta di una cricca ("frattura fragile "). Estrapolando i risultati ottenuti ad alta temperatura [3] è possibile prevedere la vita utile dei tubi alla temperatura di esercizio. Purtroppo queste prove sono molto costose per via della lunga durata (1-2 anni).

La meccanica della frattura (MF) può fornire un approccio alternativo al problema. In particolare è possibile caratterizzare il comportamento a frattura del materiale attraverso prove di laboratorio. Una volta note le proprietà del materiale è possibile prevedere la vita utile di un qualunque manufatto (ad esempio un tubo in pressione). Passoni et al. [4] hanno studiato i due materiali oggetto anche del presente lavoro effettuando prove di creep ad alta temperatura della durata di alcune settimane, dunque assai più brevi delle prove sui tubi discusse in precedenza. Gli autori hanno poi applicato un modello analitico per prevedere la vita utile di tubi in pressione. Il confronto delle previsioni con le curve di cedimento riportate sulla norma ISO 12230 [5] ha mostrato risultati promettenti.

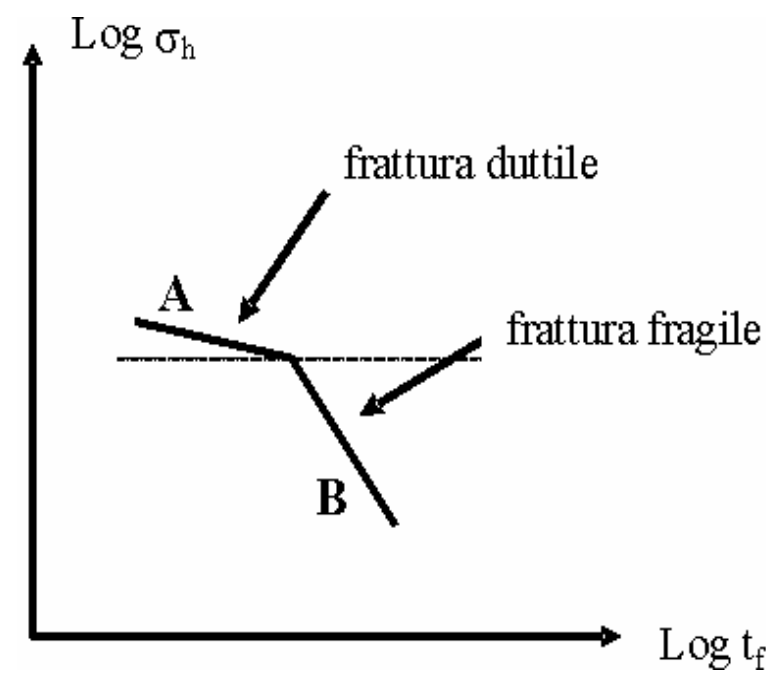

Figura 1. Andamento schematico della relazione tra sforzo circonferenziale e tempo di vita utile per un tubo in pressione realizzato in materiale polimerico.

Andena et al. [6] hanno invece studiato il comportamento a frattura degli stessi due materiali a temperatura ambiente. Dal punto di vista fenomenologico si è evidenziata la presenza di due diversi meccanismi di frattura, uno dei quali responsabile di una parziale instabilità durante la propagazione. Si sono poi determinate le proprietà all'innesco in accordo con la MF ed è stato applicato un approccio numerico di modellazione coesiva $[7,8]$. Lo strumento di calcolo ha prodotto risultati in buon accordo con i dati sperimentali relativi all'innesco. Si è tentato di simulare anche la propagazione ma il modello coesivo adottato non è abbastanza sofisticato per riprodurre adeguatamente una fenomenologia complessa quale quella osservata nell'i-PB1.
Nel presente lavoro si è nuovamente fatto ricorso alla MF per caratterizzare il comportamento dell'i-PB1 durante la propagazione. Si sono svolte prove di laboratorio a diverse temperature di durata variabile da alcuni secondi a poco più di un'ora: questi tempi sono assai più brevi di quelli richiesti dalle prove di creep o da quelle sui tubi. In questo modo è stato possibile effettuare previsioni adottando un modello analogo a quello utilizzato in [4] e verificarne l'attendibilità attraverso il confronto con dati sperimentali ottenuti da prove su tubi.

\section{ASPETTI TEORICI}

La MF identifica nel fattore di intensificazione degli sforzi $K_{I}$ la grandezza che esprime l'intensità del campo di sforzi all'apice di un difetto, indipendentemente dalla configurazione geometrica del corpo in esame. Il suffisso primo si riferisce al fatto che si sta qui considerando il modo di frattura di apertura, solitamente più critico per la maggior parte delle applicazioni; d'ora in avanti il pedice verrà omesso. $K$ è funzione dello sforzo nominale $\sigma$ (conseguente al carico applicato al contorno, la pressione interna nel caso di un tubo) e dell'entità del difetto $a$, attraverso un fattore di forma che tiene conto della geometria effettiva:

$$
K=Y \cdot \sigma \cdot \sqrt{a}
$$

Diversi autori tra cui Williams [9] e Schapery [10] hanno suggerito possibili approcci per applicare la MF a materiale viscoelastici, quali i polimeri. In particolare Williams ha mostrato, sotto opportune ipotesi semplificative, la validità delle seguenti relazioni tra il fattore di intensificazione degli sforzi $K$, il tempo di innesco $t_{i}$ e la velocità di avanzamento della cricca $\dot{a}$ :

$$
\begin{aligned}
& t_{i}=B \cdot K^{-p} \\
& \dot{a}=A \cdot K^{q}
\end{aligned}
$$

in cui $A, B, p$ e $q$ sono proprietà del materiale ed in generale dipendono dalla temperatura di prova. Esse possono essere determinate a partire da prove di frattura condotte su campioni opportunamente intagliati.

Schapery [11] ha inoltre dimostrato che $\dot{a}$ dipende dal valore istantaneo di $K$ ma non dai suoi valori passati e ciò permette di applicare la relazione (3) a qualunque storia di carico.

È così possibile formulare una previsione della vita utile $t_{f}$ di un tubo in pressione avente spessore di parete $s$ :

$t_{f}=t_{i}+t_{p}=B \cdot K_{0}^{-p}+\int_{a_{0}}^{s} \frac{d a}{A \cdot K^{q}}$

Nella (4) $t_{p}$ rappresenta il tempo necessario perché dopo l'innesco della frattura un difetto di dimensione iniziale $a_{0}$ divenga passante. $K_{0}$ è il valore del fattore di intensifi- 
cazione degli sforzi (corrispondente ad $a_{0}$ ) che rimane costante durante la fase di innesco della frattura, essendo costante la pressione (e dunque lo sforzo) nel tubo.

\section{DETTAGLI SPERIMENTALI}

Si sono studiati due polibuteni per tubi forniti da Basell Polyolefins, aventi diverso grado di isotatticità e dunque di cristallinità. I due materiali sono gli stessi studiati in [4] e in analogia al precedente lavoro verranno nel seguito indicati come PB1 e PB2 (quest'ultimo con cristallinità maggiore).

Entrambi, forniti sotto forma di granuli, sono stati stampati a compressione in lastre di dimensione 170x120x10 $\mathrm{mm}$. In seguito al raffreddamento da fuso l'i-PB1 cristallizza in forma II, avente simmetria tetragonale. Questa forma è metastabile ed a temperatura ambiente transisce spontaneamente nella forma I, che possiede un reticolo esagonale. Onde permettere il completamento della transizione i campioni sono stati tagliati dalle lastre e fresati dopo un'attesa di almeno 15 giorni dallo stampaggio [4].

Per studiare la propagazione in modo I si è scelto di utilizzare due configurazioni di prova, double cantilever beam (DCB) e single edge notched bending (SENB), mostrate rispettivamente in Fig. 2 e Fig. 3.

Le dimensioni dei campioni per entrambe le geometrie sono indicate in Tab 1 . Sono stati praticati intagli con raggio di curvatura all'apice pari a $13 \mu \mathrm{m}$.

In entrambi i casi sono state realizzate scanalature laterali per guidare l'avanzamento della frattura lungo il piano desiderato. Le scanalature, non mostrate in figura, sono state generate alla fresa con un utensile avente profilo a $\mathrm{V}$ di $60^{\circ}$ procedendo su ciascun lato dei campioni fino ad una profondità pari al 10\% dello spessore nominale.

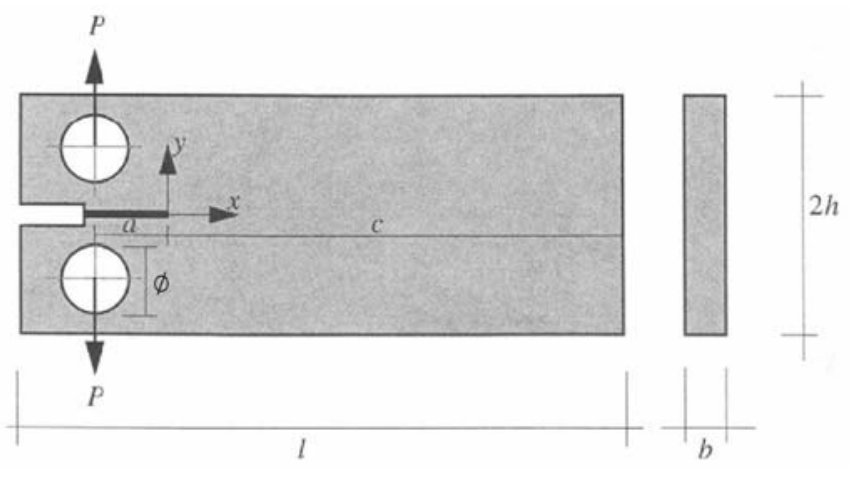

Figura 2. Provino DCB
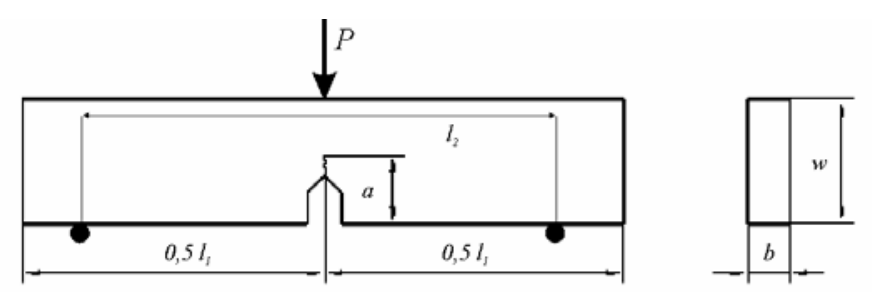

Figura 3 - Provino SENB

\begin{tabular}{cc|cc}
\hline \multicolumn{2}{c|}{ DCB } & \multicolumn{2}{c}{ SENB } \\
\hline $2 \mathrm{~h}$ & $45 \mathrm{~mm}$ & $\mathrm{w}$ & $20 \mathrm{~mm}$ \\
\hline $\mathrm{l}$ & $165 \mathrm{~mm}$ & $\mathrm{l}_{1}$ & $88 \mathrm{~mm}$ \\
\hline $\mathrm{a}$ & $60 \mathrm{~mm}$ & $\mathrm{a}$ & $10 \mathrm{~mm}$ \\
\hline $\mathrm{b}$ & $10 \mathrm{~mm}$ & $\mathrm{~B}$ & $10 \mathrm{~mm}$ \\
\hline$\varnothing$ & $7.5 \mathrm{~mm}$ & $\mathrm{l} 2$ & $80 \mathrm{~mm}$ \\
\hline $\mathrm{c}$ & $90 \mathrm{~mm}$ & &
\end{tabular}

Tabella 1. Dimensioni dei campioni DCB e SENB

Le prove di frattura sono state condotte su un dinamometro elettromeccanico Instron. Si sono utilizzate velocità di spostamento imposte di 1,10 e $100 \mathrm{~mm} / \mathrm{min}$. Il dinamometro equipaggiato con una camera termostatica ha permesso di svolgere le prove alle temperature di 23, 50, 70 e $90^{\circ} \mathrm{C}$. Per ogni condizione di prova (configurazione, velocità e temperatura) si sono effettuate almeno due ripetizioni. La lunghezza di cricca è stata rilevata attraverso misure ottiche effettuate riprendendo con una telecamera i campioni, sui quali era stato posto un opportuno riferimento di calibrazione.

\section{RISULTATI}

Durante le prove si è osservata una parziale instabilità nella propagazione della frattura. Questo fenomeno si manifesta anche nell'aspetto molto irregolare che le curve carico-spostamento per i due materiali hanno successivamente all'innesco. A titolo di esempio in Fig. 4 sono riportate le curve relative alle prove SENB a $23^{\circ} \mathrm{C}$ e 1 $\mathrm{mm} / \mathrm{min}$, unitamente ai valori dei parametri critici di MF all'innesco. Si osserva che il PB2, caratterizzato da un maggior grado di isotatticità, è più tenace del PB1.

Attraverso le riprese effettuate durante le prove è stato possibile osservare la peculiare fenomenologia di frattura di questi materiali (v. Fig. 5). Davanti all'apice della cricca si formano una o più regioni in cui il materiale è fortemente stirato. La frattura procede attraverso una lacerazione continua di queste zone (propagazione stabile) le quali di tanto in tanto cedono bruscamente (propagazione instabile). Questo secondo meccanismo determina discontinuità nella propagazione della cricca, visibile in Fig. 6. A questo fenomeno è associata la presenza di aree scure e lisce sulle superfici di frattura il cui aspetto differisce molto da quello delle regioni in cui la propagazione è avvenuta in modo stabile [6].

Dalla Fig. 6 si può però osservare come il fattore di intensificazione degli sforzi si mantenga su valori pressoché costanti, nonostante la velocità di avanzamento della cricca subisca variazioni considerevoli per la presenza delle discontinuità. In virtù di questo fatto si è ritenuto più significativo considerare per ogni prova un unico valore medio di $\dot{a}$ rapportandolo al valore medio di $K$ 


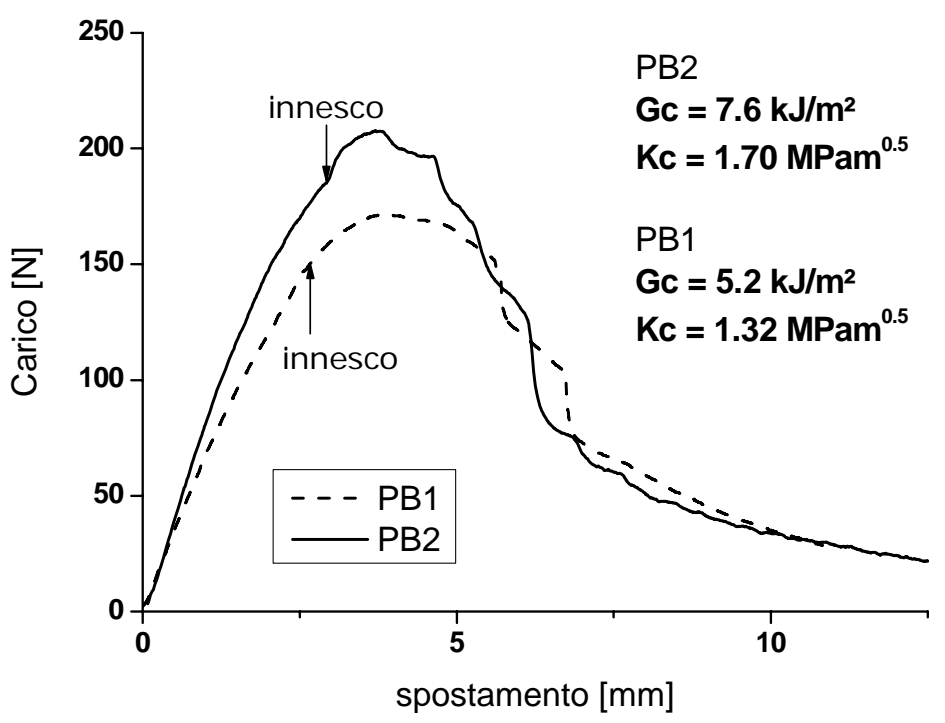

Figura 4. Risultati delle prove SENB a $23^{\circ} \mathrm{C}, 1 \mathrm{~mm} / \mathrm{min}$

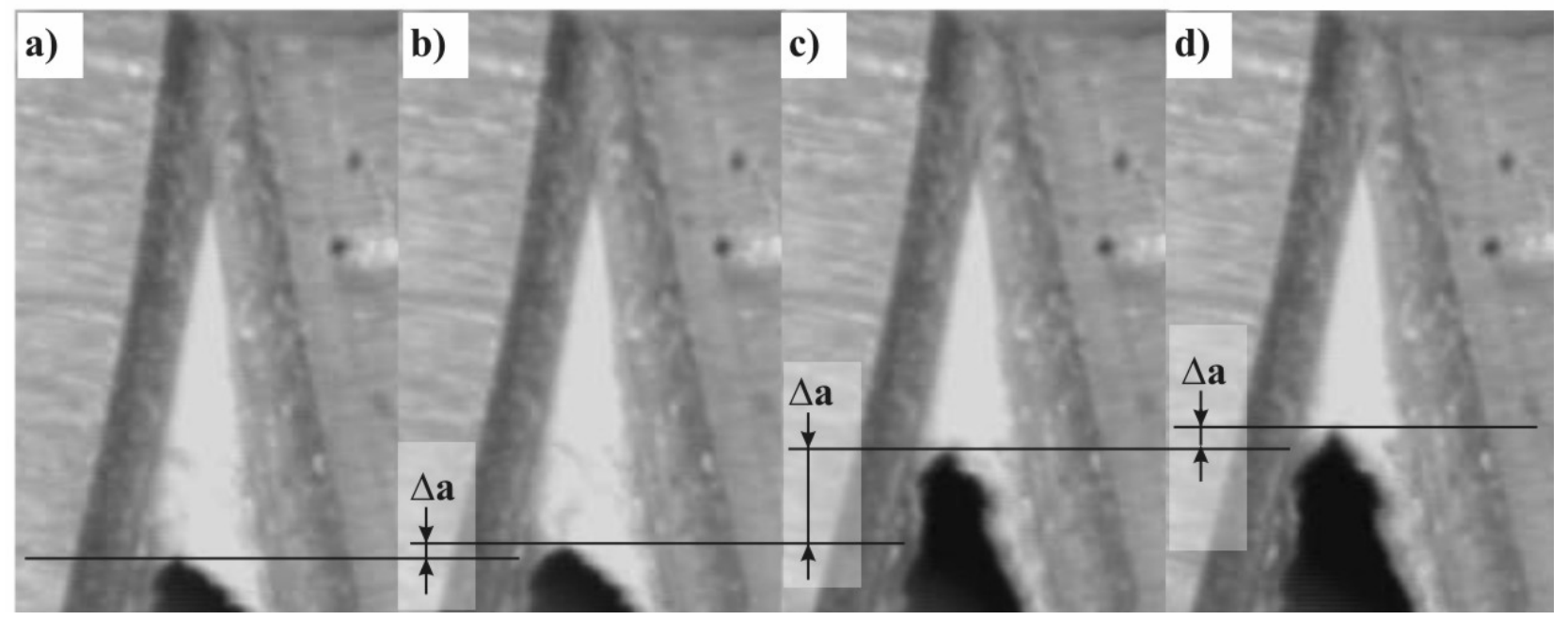

Figura 5. Meccanismi di propagazione stabile/instabile in una prova SENB su PB2 a $50^{\circ} \mathrm{C}$ e $1 \mathrm{~mm} / \mathrm{min}$ : le immagini sono prese ad intervalli regolari di 16 s. Tra 4b e 4c si può osservare un episodio di propagazione instabile che si sovrappone a quella stabile (visibile tra 4a e 4b, tra 4c e 4d). Si può notare chiaramente la zona di materiale stirato antistante l'apice della cricca

durante la fase di propagazione, come indicato schematicamente in Fig. 6.

Il grafico in scala bilogaritmica di Fig. 7 mostra i risultati ottenuti dalle prove DCB, condotte a temperatura ambiente. L'andamento lineare ipotizzato dall'equazione (3) è confermato dai dati sperimentali. È anche possibile osservare come il PB2 oltre ad essere più tenace all'innesco offra una maggiore resistenza all'avanzamento della frattura. Per entrambi i materiali la pendenza delle curve è molto bassa, segno di una modesta influenza della viscoelasticità sul comportamento durante la propagazione.

Determinata la relazione tra $K$ e $\dot{a}$ è stato possibile applicare il modello analitico proposto in [4] per prevedere il tempo di vita di tubi in pressione e confrontarlo con i dati sperimentali disponibili. Nel modello si è considerato un difetto piano di forma semicircolare situato sulla superficie interna del tubo e giacente in un piano radiale, ammettendo che la sua forma non cambi durante la propagazione. Si sono ipotizzati un diametro interno del tubo di
$100 \mathrm{~mm}$, uno spessore di parete di $10 \mathrm{~mm}$ e un raggio iniziale del difetto di $50 \mu \mathrm{m}$. In analogia con quanto fatto in [4] nel calcolo si è trascurato il tempo di innesco, formulando quindi una previsione conservativa della vita utile del tubo.

Per validare il modello si sono confrontate le sue previsioni con dati sperimentali ottenuti da prove su tubi in pressione eseguite applicando valori di sforzo circonferenziale fino a $20 \mathrm{MPa}$. In corrispondenza di questo valore massimo di sforzo i valori di $\log K$ calcolati per il modello vanno da -0.66 (per $a \sim a_{0}$ ) fino a 0.48 (per $a \sim s$ ). Durante le prime fasi della propagazione questi valori sono molto più bassi di quelli rilevati durante le prove di laboratorio (v. Fig. 7). Si è così effettuata una estrapolazione ipotizzando valida l'equazione (3): a causa della bassa pendenza delle curve $K-\dot{a}$ si ottengono valori estremamente bassi di $\dot{a}$, dell'ordine di $10^{-30} \mathrm{~mm} / \mathrm{s}$ al limitare inferiore del campo di $K$ considerato. Ciò determina previsioni di tempi lunghissimi (nell'ordine di $10^{20} \mathrm{~h}$ ) per 


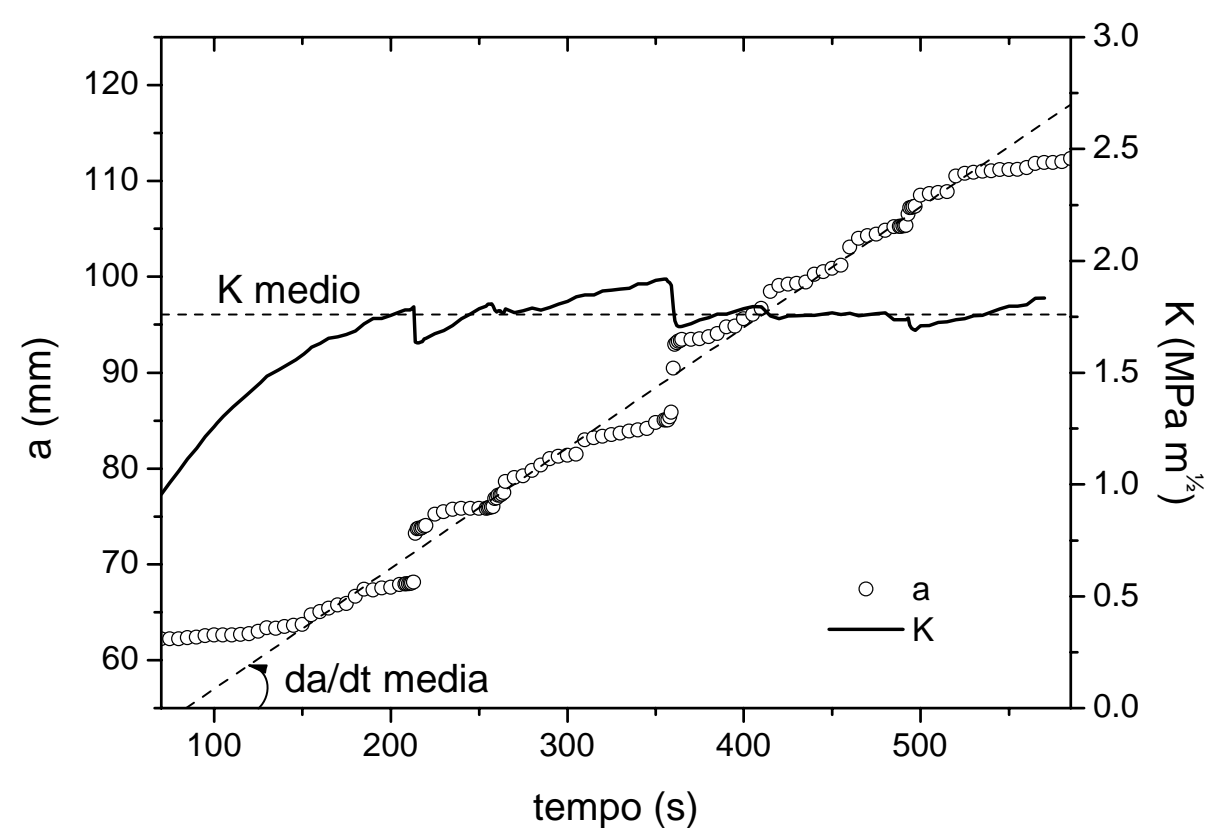

Figura 6. Andamento di K e a durante una prova DCB su PB1 a $23^{\circ} \mathrm{C}$ e $10 \mathrm{~mm} / \mathrm{min}$

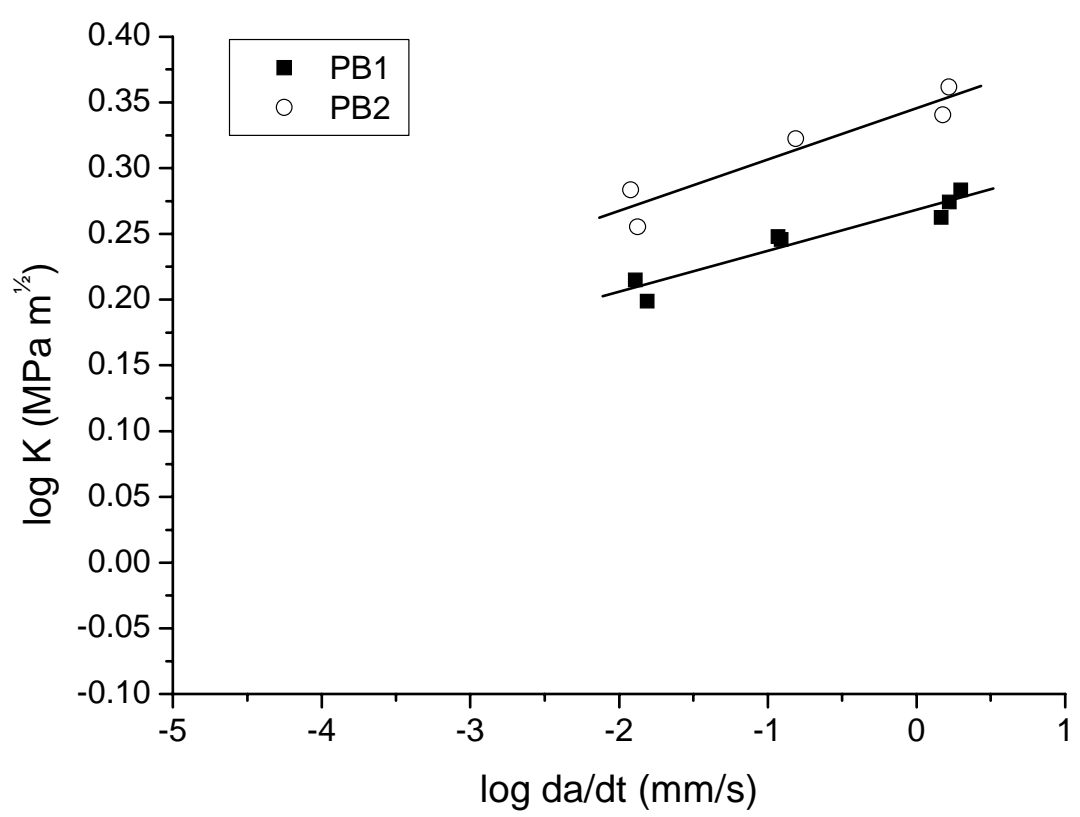

Figura 7. Risultati delle prove DCB a $23^{\circ} \mathrm{C}$ per i due materiali

osservare il cedimento del tubo, assolutamente non realistiche.

Per esplorare il campo dei valori inferiori di $K$ si sono quindi svolte, sul solo PB2, prove di frattura in configurazione SENB a temperature superiori a quella ambiente, in particolare 50,70 e $90^{\circ} \mathrm{C}$. I risultati sono mostrati in Fig. 8: si può notare un netto cambiamento nell'andamento della curva $K-\dot{a}$ all'aumentare della temperatura.

È stato applicato uno schema di riduzione dei dati secondo l'equivalenza tempo-temperatura traslando le curve ottenute a temperature diverse in modo da costruire una curva maestra ad una temperatura di riferimento (si veda ad esempio [12]). In questo modo è stato possibile ottenere una curva estesa su sei decadi di velocità di avanza- mento con una buona sovrapposizione dei dati (come mostrato in Fig. 9) alle diverse temperature. L'andamento del fattore di spostamento è pressoché lineare in un grafico di tipo Arrhenius.

Come detto l'applicazione del modello al tubo in pressione richiede l'estrapolazione dei dati sperimentali a valori di $\mathrm{K}$ inferiori: questa è stata eseguita sulla base del ramo della curva originato dai dati ad alta temperatura. In questo caso i valori di $\dot{a}$ calcolati all'inizio della propagazione sono dell'ordine di $10^{-10} \mathrm{~mm} / \mathrm{s}$, superiori di ordini di grandezza rispetto a quelli calcolati in precedenza. Le previsioni della vita utile del tubo così ottenute sono in buon accordo con i dati sperimentali disponibili sia per quanto riguarda il posizionamento delle curve in assoluto 


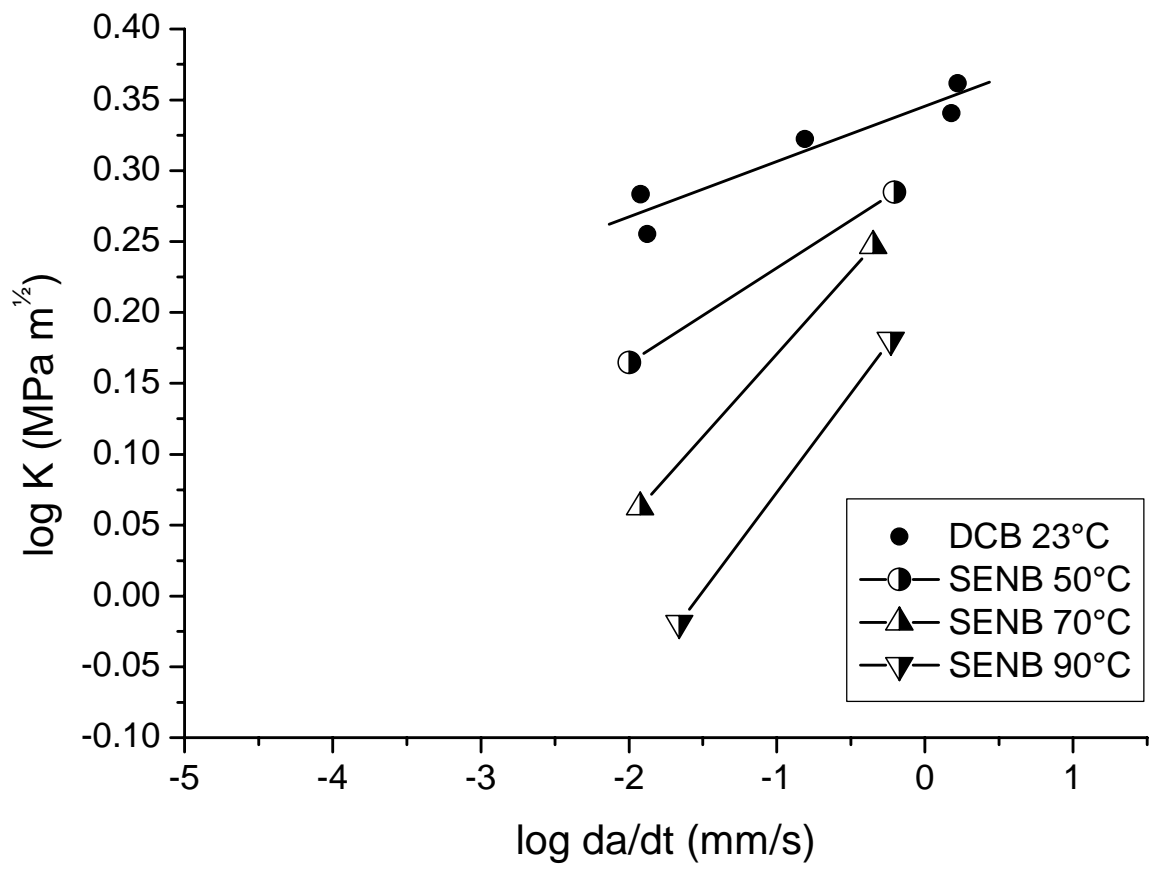

Figura 8. Risultati delle prove DCB e SENB su PB2 a diverse temperature. Per le prove SENB i punti rappresentano la media dei valori ottenuti

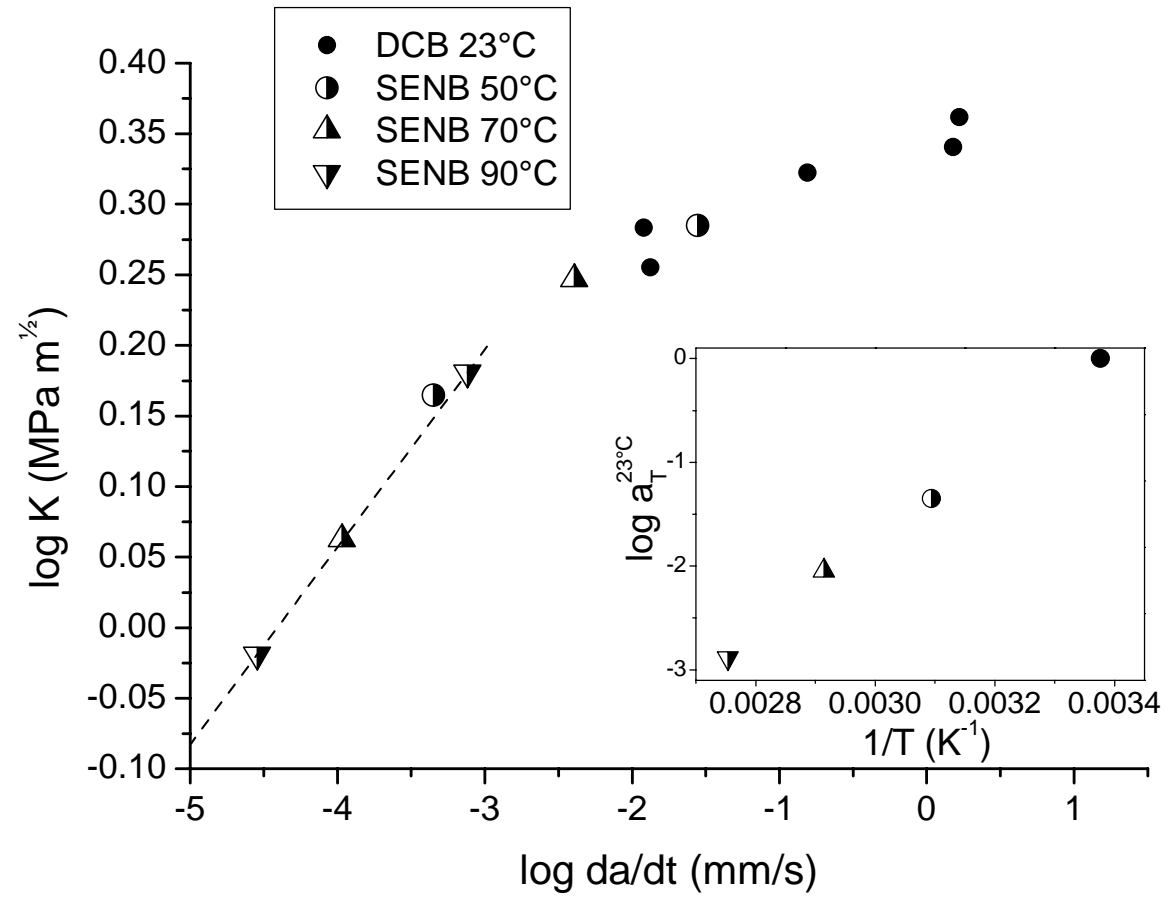

Figura 9. Curva maestra di $\log \mathrm{K}-\mathrm{da} / \mathrm{dt}$ a $23^{\circ} \mathrm{C}$ e relativo fattore di spostamento. In figura è anche indicata la retta di estrapolazione utilizzata nell’applicazione del modello del tubo.

che per l'effetto della variazione di temperatura (v. Fig. $10)$.

È da rilevare che nelle prove sui tubi si è osservata quasi esclusivamente frattura duttile mentre il modello rappresenta il comportamento del materiale durante la propagazione lenta di una cricca. Idealmente queste due situazioni corrispondono ai due distinti rami della curva mostrata in Fig. 1. Le previsioni del modello suggeriscono una transizione da comportamento duttile a fragile che dovrebbe essere osservata a tempi di poco superiori a quelli rilevati sperimentalmente: ciò potrebbe essere confermato qualora si rendessero disponibili dati relativi al cedimento di tubi in prove condotte a valori di sforzo più bassi. È bene notare che nelle previsioni del modello si è trascurato il tempo di innesco: qualora se ne tenesse conto si avrebbero curve spostate verso tempi maggiori. Inoltre le previsioni sono influenzate dalle ipotesi fatte su forma e dimensioni iniziali del difetto, che potrebbero influenzare la posizione delle curve. 


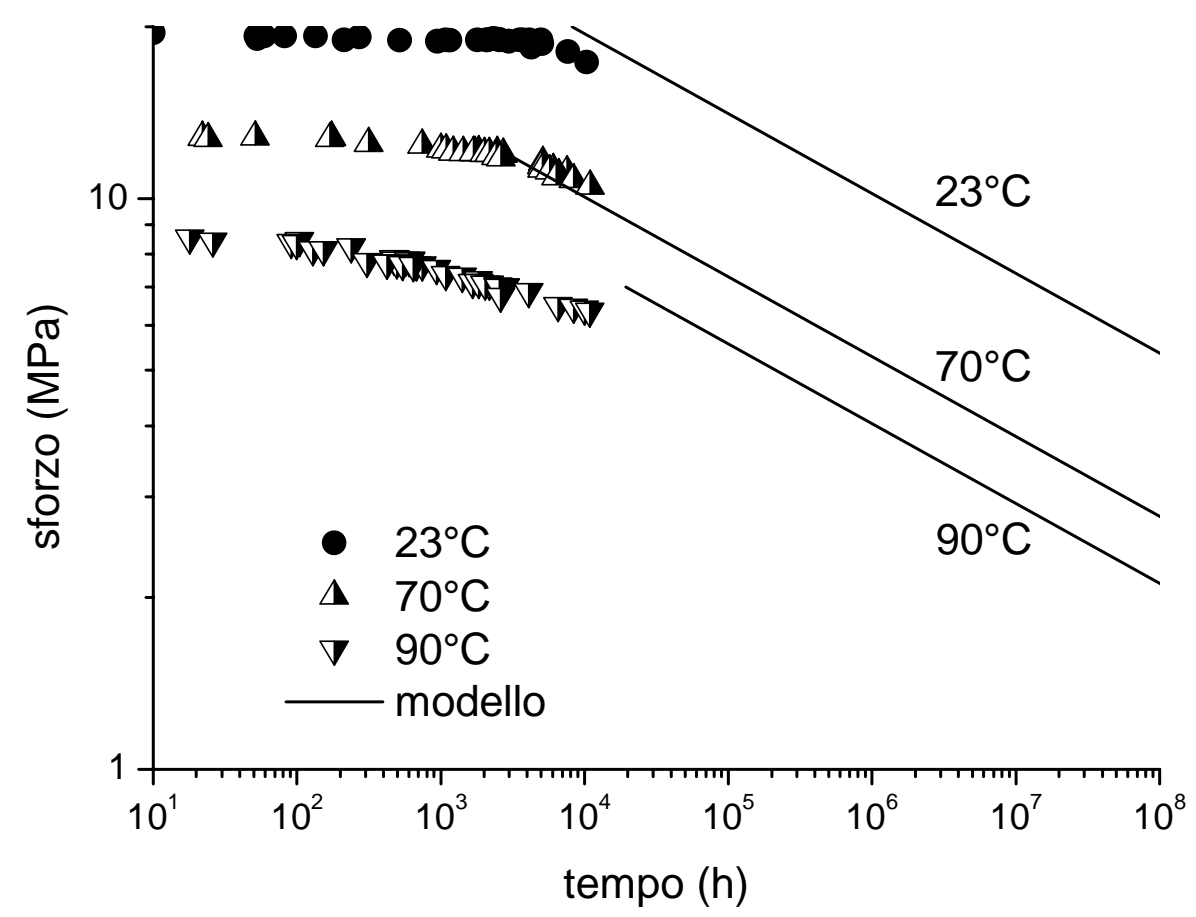

Figura 10. Confronto tra i dati sperimentali ottenuti da prove su tubi con le previsioni del modello ottenute in base ai dati mostrati in Fig. 9.

\section{CONCLUSIONI}

I risultati ottenuti hanno confermato la validità di un approccio di meccanica della frattura per la caratterizzazione di polibutene utilizzati per la realizzazione di tubi in pressione. Lo strumento della MF appare del tutto adeguato a mettere in luce differenze di comportamento a lungo termine tra materiali diversi attraverso una caratterizzazione di laboratorio estremamente più rapida ed economica di quella sul manufatto finale. Questa caratterizzazione permette inoltre l'applicazione di modelli in grado di prevedere direttamente in modo attendibile la vita utile dei tubi in pressione.

Naturalmente non si può pensare di sostituire completamente questo approccio alle prove sui tubi, soprattutto in vista dei severi controlli normativi previsti per la certificazione dei materiali per queste applicazioni. Con la MF è però possibile discriminare fra materiali diversi accelerando notevolmente lo sviluppo di nuove formulazioni. Una volta giunti alla definizione della formulazione più idonea è possibile avviare la campagna di sperimentazione sui tubi per la verifica delle previsioni della MF.

Le indagini svolte hanno confermato la complessa natura del comportamento a frattura del polibutene, già messa in luce in precedenti lavori. Lo schema di riduzione tempotemperatura è stato applicato con successo per la descrizione della propagazione della frattura dell'i-PB1. Ulteriori prove sperimentali attualmente in corso potranno confermare la bontà dell'approccio.

Sviluppi futuri di sicuro interesse sono l'approfondimento dello studio della fase di innesco e un chiarimento dei diversi meccanismi di frattura in atto alle diverse temperature.

\section{RINGRAZIAMENTI}

Si ringrazia l'ing. Paolo Redondi per il prezioso supporto fornito durante l'esecuzione delle prove DCB.

\section{BIBLIOGRAFIA}

[1] AM. Chatterjee, Butene polymers. In: Encyclopaedia of Polymer Science and Engineering, 1985. p. 590.

[2] F. Azzurri, A.Flores, GC. Alfonso, FJ.Baltà Calleja, Polymorphism of isotactic poly(1-butene) as revealed by microindentation hardness. 1. Kinetics of the transformation. Macromolecules. 2002;35:9069

[3] Plastics piping and ducting systems - Determination of the long-term hydrostatic strength of thermoplastics materials in pipe form by extrapolation. ISO9080:2003(E)

[4] P. Passoni, R.Frassine, A.Pavan, Small scale accelerated tests to evaluate the creep crack growth resistance of polybutene pipes under internal pressure. Proceedings of Plastics Pipes XII Milan 2004.

[5] Polybutene (PB) pipes - Effect of time and temperature on the expected strength. ISO12230:1996(E)

[6] L. Andena, M. Rink, JG. Williams, Cohesive zone modelling of fracture in polybutene. Engineering Fracture Mechanics. 2006;73:2476-2485.

[7] S. Bianchi, A. Corigliano, R. Frassine, M. Rink, Modelling of Interlaminar Fracture Processes in Composites using Interface Elements. Compos. Sci. Technol. 2006;66:255-63.

[8] L. Andena, M. Rink, Fracture of rubber-toughened poly (methyl methacrylate): measurement and study of 
cohesive zone parameters. Proceedings of ICF XI Turin 2005.

[9] JG. Williams, The use of fracture mechanics in design with polymers. Plasticon 81. Engineering design with plastics: principles and practice, University of Warwick. 1981.

[10] RA. Schapery, A theory of crack initiation and growth in viscoelastic media. I. Theoretical development. International Journal of Fracture. 1975;11:141-159.
[11] RA. Schapery, A theory of crack initiation and growth in viscoelastic media. III. Analysis of continuous growth. International Journal of Fracture. 1975;11:549562.

[12] P. Mariani, R. Frassine, M. Rink, A. Pavan, Viscoelasticity of Rubber-Toughened Poly(Methyl Methacrylate). Part II: Fracture Behavior. Polymer Engineering and Science. 1996;36:2758-2764 